\title{
TTR
}

Traduction, terminologie, re?daction

\section{Les manuels de traduction : essai de classification}

\section{Jean Delisle}

Volume 5, numéro 1, 1er semestre 1992

La pédagogie de la traduction : questions actuelles (1) et Miscellanées traductologiques

URI : https://id.erudit.org/iderudit/037105ar

DOI : https://doi.org/10.7202/037105ar

Aller au sommaire du numéro

Éditeur(s)

Association canadienne de traductologie

ISSN

0835-8443 (imprimé)

1708-2188 (numérique)

Découvrir la revue

Citer cet article

Delisle, J. (1992). Les manuels de traduction : essai de classification. TTR, 5(1),

17-47. https://doi.org/10.7202/037105ar d'utilisation que vous pouvez consulter en ligne.

https://apropos.erudit.org/fr/usagers/politique-dutilisation/ 


\section{Les manuels de traduction: essai de classification}

\section{Jean Delisle}

En 1975, J. P. Barra écrivait dans Taal Taalkunde Vertaalkunde:

Malgré l'accroissement considérable d'instituts spécialisés dans la formation de traducteurs depuis la deuxième guerre mondiale et l'intérêt croissant porté à l'étude de la traduction en tant que discipline scientifique, il est assez navrant de devoir constater qu'il n'existe aucune étude complète sur l'enseignement de la traduction et de sa (ses) technique(s) ${ }^{1}$.

Pour sa part, la première présidente de l'Association canadienne de traductologie, Judith Woodsworth, constatait dans la revue TTR:

At a time of steady advances in the field of translation studies and of an increasing awareness of the importance of translation pedagogy, it is surprising that there has not been more work done to produce effective teaching tools. ${ }^{2}$

Il est vrai que nous disposons d'assez peu d'instruments pédagogiques adaptés aux exigences particulières de la formation des futurs traducteurs professionnels. Je pense pouvoir dire que c'est le cas

1. «Quelques considérations sur l'enseignement de la traduction», p. 9.

2. Compte rendu de l'ouvrage de Pamela Russell, How to Write a Précis, p. 131. 
en théorie de la traduction, en documentation, en révision, en traduction technique, en traductique et en histoire de la traduction. Le choix est un peu plus vaste en ce qui concerne l'initiation à la traduction générale.

D'un point de vue historique, si l'on fait exception de l'opuscule qu'Étienne Dolet fit paraître à Lyon en 1540 sous le titre la Manière de bien traduire d'une langue en aultre, on peut considérer comme le premier manuel de traduction en langue française le traité de Gaspard de Tende (1618-1697) intitulé De la traduction ou Règles pour apprendre d traduire la langue Latine en langue Françoise. Son auteur consigne de nombreuses règles de traduction puisées chez divers auteurs, et organise son traité comme un véritable «manuel de traduction. $\aleph^{3}$

Sans remonter aussi loin dans le temps, j'ai dressé, pour le domaine anglais-français, un inventaire des manuels publiés depuis la Deuxième Guerre mondiale, soit depuis l'apparition de la majorité des grandes écoles de traduction dans le monde. J'ai recensé pas moins d'une cinquantaine de titres, indépendamment de leur valeur pédagogique. Ces ouvrages sont utilisés au niveau universitaire, mais pas exclusivement. Plus de la moitié d'entre eux sont postérieurs à 1980. Deux facteurs expliqueraient cette prolifération des manuels de traduction: au cours des douze dernières années, on a assisté à la consolidation des programmes de traduction et à la réhabilitation des exercices de traduction dans les cours de langue ${ }^{4}$.

Les titres de la liste ci-dessous, qui n'a aucune prétention d'exhaustivité, sont classés par ordre chronologique. Ils incluent quatre

3. Paul Horguelin, Anthologie de la maniere de traduire, p. 100.

4. «ll faut reconsidérer le rôle de la traduction dans l'apprentissage [d'une langue étrangère]. Les méthodologies qui se développent depuis les années 70, qu'elles soient appelées "fonctionnellesnotionnelles" ou plus largement "communicatives", vont dans ce sens. [...] elles avancent une théorie de l'apprentissage où la langue maternelle retrouve sa place et, de ce fait, réhabilitent la comparaison entre les langues et la traduction.» Elisabeth Lavault, Fonctions de la traduction en didactique des langues, p. 16. 
rééditions et deux titres traduits. Un [C] indique un ouvrage canadien ou publié à l'étranger par des Canadiens, tandis que les symboles $[\mathrm{F}]$ et [A] signifient respectivement la France et l'Angleterre.

\section{LES MANUELS DE TRADUCTION \\ Domaine anglais-français}

(1952-1992)

(1952) J.-P. VINAY (éd.), Traductions. Mélanges offerts en mémoire de Georges Panneton [C]

(1958) J.-P. VINAY et J. DARBELNET, Stylistique comparée du français et de l'anglais [C]

(1968) J.-P. VINAY et J. DARBELNET, Cahier d'exercices / Work Book $n^{\circ}$ 1. (Complément de la Stylistique comparée...) [C]

(1968) L. BONNEROT (et al.), Chemins de la traduction [F]

(1968) C. BRUNETEAU et J. M. LUCCIONI, Guide de la version anglaise [F]

(1969) J. MAILLOT, La Traduction scientifique et technique [F]

(1970) M. CHARLOT, Guide du thème anglais [F]

(1971) A. CULIOLI (et al.), Versions anglaises [F]

(1972) J.-P. VINAY et J. DARBELNET, Cahier d'exercices / Work Book $n^{\circ}$

2. (Complément de la Stylistique comparée...) [C]

(1972) I. DE BUISSERET, Guide du traducteur [C]

(1972) C. BRUNETEAU et J. M. LUCCIONI, Nouveau guide de la version anglaise $[\mathrm{F}]$

(1973) F. FULLER, A Handbook for Translators [A]

(1974) D. GOUADEC, Comprendre et traduire [F]

(1975) I. DE BUISSERET, Deux langues, six idiomes. (Nouvelle édition du. Guide du traducteur, 1972) [C]

(1975) P. BACQUET, Initiation au thème anglais [F]

(1977) P. HORGUELIN et J.-P. BÉNARD, Pratique de la traduction. Version générale (+ Corrigé) [C]

(1978) P. HORGUELIN, Pratique de la révision (+ Corrigé) [C]

(1978) G. Vitale (et al.), Guide de la traduction appliquée. (Tome 1 Version) [C]

(1980) J. DELISLE, L'Analyse du discours comme méthode de traduction. (+ Livre du maître) [C]

(1980) B. HOSINGTON et P. HORGUELIN, A Practical Guide to Bilingual Revision [C]

(1980) G. VITALE (et al.), Guide de la traduction appliquée. (Tome 2 Thème) $[\mathrm{C}]$ 
(1980) M. BALLARD, La Traduction de l' anglais. Théorie et pratique. Exercices de morphosyntaxe [F]

(1981) J. GUILLEMIN-FLESCHER, Syntaxe comparée du français et de l'anglais. Problèmes de traduction [F]

(1981) A. DUFF, The Third Language. Recurrent Problems of Translation into English [A]

(1982) M. CHARLOT (et al.), Pratique du thème anglais [F]

(1984) F. FULLER, The Translator's Handbook. (Nouvelle édition de $A$ Handbook for Translators, 1973) [A]

(1985) F. GRELLET, "The word against the word". Initiation à la version anglaise [F]

(1985) P. HORGUELIN, Pratique de la révision. (Nouvelle édition) [C]

(1985) Guide du réviseur, Bureau de la traduction. [C]

(1986) C. TATILON, Traduire: Pour une pédagogie de la traduction [C]

(1986) C. BEDARD, La Traduction technique. Principes et pratique [C]

(1986) A. PETTON, La Version anglaise expliquée, technique et entrainement $[\mathrm{F}]$

(1987) M. BALLARD, La Traduction de l'anglais au français [F]

(1987) H. VAN HOOF, Précis pratique de traduction médicale [F]

(1987) C. BÉDARD, Guide d'enseignement de la traduction technique. (+ Solutions des exercices) [C]

(1987) H. CHUQUET et M. PAILLARD, Approche linguistique des problemes de traduction $[\mathrm{F}]$

(1988) P. FOURNIER, Language to Language. Beginning Translation. [C]

(1988) P. NEWMARK, A Textbook of Translation [A]

(1988) M. BALLARD (et al.), Manuel de version anglaise [F]

(1989) J. DELISLE, Translation: An Interpretive Approach. (Traduction de L'Analyse du discours...) [C]

(1989) H. VAN HOOF, Traduire l' anglais. Théorie et pratique [F]

(1989) P. GUIVARC'H et C. FABRE, A Companion to Economic Translation $[\mathrm{F}]$

(1989) A. DUFF, Translation [A]

(1989) L. POLLAK, La Traduction sans peur... et sans reproche. Cours $d^{\prime}$ initiation à la version [C]

(1990) G. HARDIN et C. PICOT, Translate: Initiation à la pratique de la traduction $[\mathrm{F}]$

(1990) C. et J. DEMANUELL, Lire et traduire [F]

(1990) H. CHUQUET, Pratique de la traduction (+ Corrigé) [F]

(1990) V. WATSON RODGER, Apprendre à traduire (+ Livre du maître) [C]

(1991) F. GRELLET, Apprendre à traduire. Typologie d'exercices de traduction $[\mathrm{F}]$ 
Dans la suite de cet article, je ne décrirai pas en détail chacun de ces 49 manuels. Je tenterai plutôt de les classer en fonction de leurs objectifs généraux et de leur orientation pédagogique. En établissant ce classement, j'avais en vue les besoins particuliers des enseignants des écoles professionnelles de traduction. Cette précision est très importante comme nous le verrons plus loin. Au préalable, six observations générales s'imposent.

1) Sauf deux exceptions (M. Ballard, 1988 et P. Newmark, 1988), les auteurs de manuels de traduction semblent réticents à faire figurer le mot «manuel» ou «textbook» dans le titre de leurs ouvrages. Ils ont recours de préférence à des formules telles que «Guide de», «Pratique de», «Initiation à,, «Précis de», «Apprendre à». Craignent-ils de faire trop scolaire? On trouve pourtant au niveau universitaire de nombreux manuels d'économie, de comptabilité, d'anglais, de philosophie, etc. J'ai personnellement tendance à y voir l'indice que les objectifs d'apprentissage des cours pratiques de traduction, scolaire ou professionnelle, sont mal définis et que, par conséquent, cet enseignement se prête encore mal à un enseignement structuré, méthodique et progressif.

2) Les auteurs de manuels de traduction n'établissent pas tous une nette distinction entre, d'une part, la «pédagogie de la traduction», c'est-à-dire la formation de traducteurs professionnels s'adressant à des candidats qui sont censés au départ avoir une bonne connaissance des langues et, d'autre part, la «traduction pédagogique», c'est-à-dire l'utilisation d'exercices scolaires de traduction visant à faire acquérir une langue étrangère. Sur le plan pragmatique, l'enseignement de la traduction en tant qu'activité à part entière doit, à mon sens, faire l'économie des considérations grammaticales et de la description élémentaire des langues. Autre signe de cette absence de distinction, bon nombre de manuels font une part égale à la version et au thème: Hardin (1990), Watson Rodger (1990), Demanuelli (1990), Chuquet (1990).

3) À partir de 1980 commencent à apparaître sur le marché des manuels qui ne sont plus de simples recueils de textes à traduire, sans que ce "genre» disparaisse pour autant. Ces ouvrages sont plus spécifiquement adaptés à la formation de traducteurs professionnels et tendent à se démarquer des méthodes d'apprentissage des langues. 
Citons, entre autres, les contributions de Delisle (1980), Horguelin (1978; 1985), Bédard (1986), Van Hoof $(1987 ; 1989)$ et Guivarc'h (1989).

4) Rares sont les auteurs qui font reposer leur méthode sur des présupposés théoriques clairement énoncés. Pourtant, la conception qu'un auteur a du processus de la traduction conditionne l'ensemble de sa stratégie pédagogique et a une incidence directe sur les contenus de son enseignement. Autrement dit, ses postulats théoriques déterminent l'organisation fondamentale de son manuel. Ainsi, lorsque Daniel Gouadec écrit «Quand [l'étudiant] sait l'anglais, il sait traduire»', il implique que la traduction ne s'enseigne pas ou que cet enseignement se confond avec celui d'une langue seconde. Sa stratégie d'enseignement se remènera donc à enseigner la langue seconde. Lorsque, pour sa part, Henri Van Hoof affirme «Traduire, c'est comparer ${ }^{6}$, il révèle sa conception de la traduction. On ne s'étonne donc pas que sa méthode repose précisément sur la comparaison de segments de langue en fonction d'un certain nombre de catégories. En revanche, si un auteur, et c'est mon cas, considère qu'en tant qu'opération intellectuelle, traduire c'est d'abord interpréter le sens $d^{\prime}$ un message et manier le langage porteur du sens dans une situation précise de communication, il optera plutôt pour une stratégie pédagogique mettant en oeuvre des exercices d'analyse du sens en contexte plutôt que des exercices essentiellement comparatifs.

5) Plusieurs manuels s'adressent à un public très large et souvent mal défini et visent à répondre tout autant aux besoins des candidats-traducteurs des écoles de traduction qu'à ceux des étudiants en langues vivantes. Françoise Grellet, par exemple, estime que la majorité des exercices de son ouvrage, Apprendre d traduire, qui n'est «pas un cours [ni] une méthode de traduction», précise-t-elle, "peut préparer à la traduction littéraire tout aussi bien qu'à la traduction technique» ${ }^{7}$. Il serait étonnant que, dans une École de traduction, le

5. Comprendre et traduire, p. 3.

6. Traduire l'anglais. Théorie et pratique, p. 13.

7. Apprendre à traduire, p. 14. 
contenu du cours de traduction technique soit identique à celui du cours de traduction littéraire.

6) Enfin, les manuels manquent souvent d'homogénéité, ce qui est la conséquence inévitable du point précédent. Ainsi, dans "The word against the word". Initiation a la version anglaise, Françoise Grellet consacre des exercices à la traduction des articles (pp. 169-170) et des possessifs (p. 172). Quelques pages plus loin, elle fait comparer des traductions du monologue de Hamlet ou des pages de James Joyce et de T. S. Eliot et traduire des extraits de Dickens, Faulkner et Virginia Woolf. Ce décalage m'apparaît pour le moins incongru dans un manuel qui renferme par ailleurs d'excellentes choses. Ne doit-on pas s'attendre d'un étudiant en mesure d'évaluer et de traduire les auteurs cités qu'il connaisse le fonctionnement des articles et des possessifs en français et en anglais?

Je me limite à ces six observations générales, mais il y aurait beaucoup à dire au sujet du dosage théorie/pratique, du métalangage servant à décrire les phénomènes de la traduction, de la délimitation de la matière, des orientations méthodologiques, de l'organisation de l'enseignement en salle de classe, de la nature et de la pertinence des exercices, de la gradation des difficultés, de l'accroissement des exigences à l'égard des performances, du choix des textes, etc. Tous ces aspects méritent qu'on s'y attarde si l'on veut améliorer nos instruments de formation et faire progresser la pédagogie de la traduction.

Ces remarques préliminaires étant faites, j'en arrive à l'essentiel de mon propos: la classification des manuels de traduction. J'ai classé en sept catégories les 49 ouvrages énumérés précédemment. Chacun d'eux ayant ses qualités et ses défauts, il ne s'agira pas de porter des jugements de valeur et encore moins de jeter des anathemes. Il est toujours facile de trouver des contradictions dans les manuels, surtout ceux des autres! J'ai tenté de regrouper le plus objectivement possible ces titres en fonction de leurs principales caractéristiques. Je suis conscient que d'autres classifications sont envisageables. Les sept subdivisions retenues sont les suivantes: 
1. LES NOTES D'UN TRADUCTEUR DE MÉTIER

2. LES RECUEILS DE TEXTES (ANNOTÉS OU NON)

3. LA RÉVISION DIDACTIQUE

4. LA DEMARCHE COMPARATIVE

5. L'APPROCHE LINGUISTIQUE

6. LES FICHES DE TRAVAIL ET CAHIERS D'EXERCICES

7. L'ENSEIGNEMENT PAR OBJECTIFS D'APPRENTISSAGE

\section{CLASSIFICATION}

\section{Les notes d'un traducteur de métier}

Cette subdivision regroupe les contributions de J. Maillot (1969), I. de Buisseret (1972; 1975), F. Fuller (1973; 1984) et A. Duff (1981) ${ }^{8}$. Dans ce genre d'ouvrages, un traducteur chevronné livre son expérience professionnelle en consignant par écrit les difficultés ponctuelles qu'il a relevées dans ses travaux de traduction. Aucun de ces ouvrages ne proposent de textes à traduire. Toutefois, de nombreux exercices figurent dans le livre d'Irène de Buisseret.

Dans cette méthode, que l'on peut qualifier de «symbiotique», l'apprenti-traducteur apprend par imitation et déduction. À partir d'exemples concrets, parfois commentés, il doit lui-même déduire les règles de traduction et apprendre à éviter les pièges tendus par la langue de départ. Ces manuels ne sont pas élaborés en fonction d'un cadre théorique défini, mais d'une réflexion sur la pratique du métier. Ils sont plus ou moins des catalogues de faits de langue et de traduction, dont le classement est personnel à l'auteur.

8. On peut aussi ranger dans cette catégorie les mélanges édités par J.-P. Vinay en 1952. Bien que cet ouvrage ne soit pas l'œuvre d'une seule plume et qu'il reproduise le texte de conférences présentées devant les diplômés de l'Institut de traduction (1942-1965), il marie théorie et pratique et a servi pendant plusieurs années de manuel d'enseignement à l'Université de Montréal. 
Ces publications ont leur utilité, car les traducteurs ne disposent pas de beaucoup de façons de transmettre leur expérience professionnelle. Je trouve par conséquent quelque peu sévère le jugement sans appel de Georges Mounin à propos de Deux langues, six idiomes: «c'est, dit-il, le modèle de livre à ne plus écrire sur la traduction»". Il est vrai que la salle de cours a ses exigences et que l'apprentissage «intuitif» des faits de traduction cadre assez mal avec un enseignement universitaire structuré. Disons à la décharge de $G$. Mounin, qu'en formulant ce jugement, il dénonçait la trop grande part d'empirisme qui caractérise les ouvrages de ce genre et l'absence de cadre théorique et linguistique sous-tendant l'abondance des faits de traduction colligés ${ }^{10}$.

\section{Les recueils de textes (annotés ou non)}

Sous cette rubrique, qui regroupe le plus grand nombre de manuels, j'ai réuni les ouvrages de L. Bonnerot (1968), C. Bruneteau (1968; 1972), M. Charlot (1970), A. Culioli (1971), D. Gouadec (1974), P. Bacquet (1975), P. Horguelin (1977), G. Vitale (1978; 1980), A. Petton (1986), M. Ballard (1988), P. Guivarc'h (1989), L. Pollak (1989), H. Chuquet (1990), H. Hardin (1990) et C. Demanuelli (1990).

On peut dire que les recueils de textes constituent la méthode classique d'enseignement de la traduction. Les textes colligés par les auteurs sont soit des extraits d'oeuvres littéraires, soit des textes dits pragmatiques. Les manuels conçus surtout pour l'apprentissage de la langue renferment le plus souvent des textes littéraires. Dans les écoles professionnelles, la tendance est de proposer un éventail de textes pragmatiques puisés dans différents secteurs d'activité (économie, administration, politique, publicité, etc.) afin de préparer plus

9. «Pour une pédagogie de la traduction», Cuadernos de traducción e interpretación, p. 15.

10. Remarquons que les trois premiers ouvrages cités - Maillot (1969), de Buisseret (1972) et Fuller (1973) - sont parus au tournant des années 70, à une époque où la théorie et la pédagogie de la traduction étaient encore à leurs débuts. 
directement les candidats-traducteurs aux réalités du marché du travail. Les contributions de P. Horguelin (1977), G. Vitale (1978; 1980) et de P. Guivarc'h (1989) sont des exemples de ces manuels adaptés aux besoins des futurs traducteurs de métier. Mais les avis demeurent partagés quant à l'utilisation de textes littéraires ou pragmatiques pour initier à la pratique de la traduction ${ }^{11}$.

Autre caractéristique, ces recueils proposent soit des textes nus, soit des textes assortis d'annotations, soit le plus souvent une combinaison des deux. Les notes et commentaires sont destinés à éclairer la compréhension du texte ou à faire réfléchir sur les solutions retenues ${ }^{12}$. À cet égard, l'ouvrage d'Hélène Chuquet (1990) peut sûrement être considéré comme un modèle du genre.

Le caractère «dirigiste» de certaines annotations peut parfois étonner. Voici un exemple: «and: commencer une nouvelle phrase» ${ }^{13}$. Plus discutable encore est le recours dans les annotations à certaines catégories descriptives de la Stylistique comparée du français et de l'anglais au moment de la recherche d'une équivalence de traduction. Ainsi, la note «borne: transposition verbe/locution substantivale» ${ }^{14}$

11. C. et J. Demanuelli justifient en ces termes le choix exclusif d'extraits littéraires: «Nous concevons très bien qu'une méthode d'initiation à la traduction choisisse d'exclure le texte littéraire pour ne retenir que des textes pragmatiques. Mais ces dermiers sont loin de ne présenter que des avantages: outre les problèmes délicats de périlinguistique civilisationnelle qu'ils posent souvent, ils nécessitent pour être bien compris des connaissances extra-linguistiques relativement étendues. [...] En revanche, tout le monde a lu des romans, et s'il est un lieu où, par delà la diversité des idiomes, on parle la même langue, c'est bien la fiction, par essence transculturelle, et toujours recommencée.» Lire et traduire, pp. 9-10.

12. Au sujet des annotations, voir Robert Larose, «le Rôle des annotations de textes dans l'enseignement de la traduction», Meta, XXIX(2), pp. 143-151.

13. G. Vitale et al., Guide de la traduction appliquée, t. I, p. 35, note 20.

14. Ibid., note 13 . 
invite l'étudiant à traduire le verbe borne par une locution substantivale. L'étudiant doit trouver laquelle. Dans un autre ouvrage, on peut lire: «and never think of it again.» / «sans en conserver le moindre souvenir.» «Modulation: cause pour l'effet» ${ }^{15}$. En renvoyant systématiquement aux catégories descriptives de la Stylistique comparée, on risque d'inculquer aux étudiants de faux réflexes de traduction. En effet, au moment où il cherche à postuler une équivalence, le traducteur ne se demande jamais «Dois-je faire une transposition verbe/locution substantivale? Dois-je procéder à une modulation cause/effet?» Cela est tout à fait contraire à sa réflexion qui procède à une analyse du contexte et des compléments cognitifs. Il faut bien savoir que toutes les catégories classificatoires de la Stylistique comparée n'interviennent pas consciemment dans le processus cognitif de la traduction ${ }^{16}$.

Par ailleurs, ces ouvrages ne proposent habituellement aucun exercice portant sur une difficulté précise de traduction. L'apprentissage ne se fait pas non plus en fonction d'objectifs clairement définis, mais plus ou moins au hasard des textes, et il est difficile de déceler une progression dans le niveau des difficultés abordées. C'est aussi une méthode «symbiotique» d'apprentissage. Elle cherche à «planter des poteaux indicateurs ${ }^{17}$, à donner des repères à l'apprenti-traducteur et, dans le meilleur des cas, à lui inculquer une technique rigoureuse d'analyse des textes. Enfin, tout aussi empirique que la précédente, cette méthode laisse peu de place à la diversification des stratégies pédagogiques, l'enseignement prenant la forme d'une succession de textes à traduire ou à commenter.

15. L. Pollak, La Traduction sans peur... et sans reproche, p. 119, note 4.

16. À côté de ces catégories établies à la suite d'une observation $a$ posteriori d'équivalences de traduction, la Stylistique comparée en renferme d'autres qui peuvent avoir une réelle utilité au moment de l'établissement d'une équivalence. C'est le cas, entre autres, des notions d'économie par évidence, d'étoffement, de caractérisation, d'adaptation, de chassé-croisé, de faux comparatif.

17. L. Bonnerot, Chemins de la traduction, p. 1. 


\section{La révision didactique}

Dans un milieu de travail, la révision n'est ni une discipline ni une profession, mais une fonction. En enseignement de la traduction, le cours de révision n'a pas pour but de préparer directement à l'exercice de cette fonction.

Il est peut-être paradoxal, mais non moins évident, a écrit Paul Horguelin, qu'un cours de révision ne vise pas à former des réviseurs - sauf exceptionnellement, s'il s'adresse à des traducteurs en exercice. [...] Les objectifs sont à la fois plus modestes et plus diversifiés. [...] Confronté à une traduction qui n'est pas la sienne [...], l'étudiant peut la décortiquer à son aise, enregistrant les passages bien rendus ou, au contraire, s'efforçant de remédier aux déficiences. Il est donc de ce fait amené à tester in vivo son apprentissage de la traduction, et à accroître aussi sa compétence $[. . .]^{18}$.

Dans un programme de traduction, le cours de révision, qui se donne habituellement en terminale, constitue un moyen de consolidation des connaissances du futur traducteur. La révision didactique (par opposition à la révision pragmatique ou professionnelle, selon la distinction établie par Paul Horguelin ${ }^{19}$ ) apparaît comme un exercice très formateur qui offre à l'étudiant de multiples occasions d'affiner son «jugement linguistique», puisque chaque correction ou amélioration doit être justifiée. Ces exercices sont un bon moyen de formation, au même titre que les cours pratiques de traduction. Les ouvrages appartenant à

18. «La révision didactique», pp. 253-254.

19. «Si la révision a pour seule fonction de rendre un texte conforme à certains critères avant de le diffuser, sans qu'il $y$ ait de communication entre le réviseur et l'auteur ou le traducteur, on parlera de révision pragmatique. Si à cette fonction s'ajoute celle du perfectionnement de rédacteurs ou traducteurs, ou bien si la révision prend la forme d'un exercice en vue de la formation d'étudiants, on dira qu'il s'agit d'une révision didactique.» Pratique de la révision. p. 10. 
cette subdivision sont ceux de P. Horguelin $(1978 ; 1980 ; 1985)$ et, à un degré moindre, le Guide du réviseur du Bureau de la traduction ${ }^{20}$.

Cette méthode présente beaucoup de points communs avec la précédente - les recueils de textes. La consolidation des connaissances acquises se fait aussi plus ou moins au hasard des textes. Les objectifs de ce cours demeurent très généraux. Certains professeurs-réviseurs, comme Brian Mossop ${ }^{21}$, s'interrogent même sur la pertinence d'un tel cours, en invoquant notamment la difficulté d'en préciser les contenus.

\section{La démarche comparative}

Au nombre des manuels privilégiant la comparaison systématique des langues figurent l'ouvrage de J.-P. Vinay et J. Darbelnet (1958), celui de J. Guillemin-Flescher (1981) et ceux de H. Van Hoof (1987; 1989). Ce qui caractérise ces ouvrages est le souci des auteurs d'établir une classification des phénomènes de traduction et de doter les études contrastives d'un métalangage. Le glossaire de la Stylistique comparée fait douze pages, celui de J. Guillemin-Flescher, plus de cent. Pour sa part, $\mathrm{H}$. Van Hoof fonde sa méthode exclusivement sur la classification de Vinay et Darbelnet.

La Stylistique comparée du français et de l'anglais, qui a ouvert le champ de l'analyse contrastive en français et en anglais, propose un classement raisonné de l'ensemble des principaux faits de traduction. La principale qualité de cet ouvrage capital n'est pas d'être une «méthode de traduction», mais un instrument d'observation et de

20. «Le Guide du réviseur a été conçu afin d'aider les réviseurs du Bureau de la traduction, anciens ou nouveaux, à s'acquitter de leur travail de tous les jours. [II s'agit d'un] recueil d'indications utiles et de conseils pragmatiques, fruit d'expériences multiples [...]» (p. iii). Bien qu'il s'adresse prioritairement à des réviseurs professionnels, ce Guide est aussi utilisé dans le cours de révision d'au moins un programme universitaire de traduction, celui de l'Université d'Ottawa.

21. «Enquête sur les cours de révision». Synthèse des résultats diffusés auprès des répondants, 22 février 1991.10 p. (Inédit) 
classification des phénomènes de traduction par rapprochement de deux systèmes linguistiques. Les catégories établies par Vinay et Darbelnet et reprises par bon nombre d'auteurs de manuels déjà cités facilitent l'étude poussée de la dissociation des langues à tous les niveaux (lexical, syntaxique, stylistique).

L'ouvrage de J. Guillemin-Flescher, Syntaxe comparée du français et de l'anglais. Problemes de traduction n'est ni une dissertation sur la traduction, ni un traité de stylistique littéraire, ni un manuel de linguistique contrastive dont l'objectif serait de faire acquérir la maîtrise de la traduction. L'auteur a tenté «de dégager les opérations qui sous-tendent l'activité langagière et la façon dont elles se réalisent en français et en anglais» ${ }^{22}$. Le roman Madame Bovary sert de corpus à l'étude des phénomènes linguistiques, dans une perspective différentielle.

Quant à $H$. Van Hoof, il affirme dans ses deux livres que «la traduction compare deux systèmes linguistiques» ${ }^{23}$ et qu'elle «est essentiellement une opération de transcodage» ${ }^{24}$. Notons que l'auteur ne précise pas ce qu'il entend exactement par ce dernier terme auquel les pédagogues et les traductologues donnent des sens les plus divers. Quoi qu'il en soit, ses ouvrages renferment, outre des textes, des paires d'équivalents disposés sur deux colonnes. Celles-ci sont chapeautées de titres tels que «Comparatif-Positif», «Ellipse des articulations» ou encore «Animisme».

Cette façon statique de présenter les faits de langue relègue au second plan toute la dynamique de la traduction de textes et détourne l'attention du travail d'interprétation des textes, caractéristique de l'opération traduisante. Comparer des équivalences est une chose, enseigner le maniement du langage en est une autre. Par maniement du langage, j'entends dégager le sens d'un texte et le réexprimer de façon idiomatique tout en évitant les interférences. Si la comparaison de

22. Avant-propos, p. vii.

23. Traduire l'anglais. Théorie et pratique, p. 11.

24. Précis pratique de traduction médicale, p. 11. 
langue intervient en pédagogie de la traduction, cet enseignement ne saurait se ramener à ce genre d'exercices, si utiles soient-ils.

La démarche comparative rend nécessaire de rappeler la distinction qui existe entre «traduire la langue» et «traduire des discours». La démarche du stylisticien comparatiste est descriptive et classificatoire - son objet étant l'analyse des équivalences résultant du processus de traduction -, tandis que la démarche du traducteur est interprétative et communicative - son objet étant la postulation des équivalences.

Dans l'allocution inaugurale qu'elle prononça à l'occasion du XII ${ }^{e}$ Congrès mondial de la FIT (Belgrade, août 1990) ${ }^{25}$, Danica Seleskovitch a très clairement illustré les trois niveaux de traduction que distingue la théorie interprétative: 1) langue, 2) parole et 3) discours/texte. Je reprends ici son excellent exemple afin de bien faire voir l'importance de maintenir cette distinction capitale en enseignement de la traduction professionnelle.

Le niveau langue est celui des mots hors contexte, polysémiques.

\begin{tabular}{||l|l|l|l|}
\hline \multicolumn{4}{|c|}{ MOTS HORS CONTEXTE } \\
\hline \hline you & vous? & tu? & \\
\hline sight & vision? & vue? & yeux? \\
\hline to urge & pousser? & insister? & demander? \\
\hline low & bas? & inférieur? & humble? \\
\hline
\end{tabular}

Le niveau parole est celui des phrases construites ou étudiées en dehors des paramètres discursifs. Leur transcodage produit des correspondances qui ne sont pas sans utilité en enseignement des langues.

25. «Traduction et traductologie». A paraître dans les Actes du congrès. 


\begin{tabular}{|c||}
\hline \multicolumn{1}{|c||}{ ÉNONCÉ ORIGINAL } \\
\hline $\begin{array}{l}\text { "In your opening remarks to the conference, Prime } \\
\text { Minister, you urged the delegations not to set their } \\
\text { sights too low." }\end{array}$ \\
\hline VERSION TRANSCODÉE \\
\hline $\begin{array}{l}\text { «Dans vos observations liminaires à la conférence, Pre- } \\
\text { mier Ministre, vous avez demandé avec insistance aux } \\
\text { délegations de ne pas fixer leurs regards trop bas.» }\end{array}$ \\
\hline
\end{tabular}

Le niveau discours suppose des compléments cognitifs, c'est-àdire des connaissances extra-linguistiques. Voici ceux que possédait l'interprète (un traducteur aurait eu besoin des mêmes compléments cognitifs): Il s'agit d'une conférence internationale tenue à Londres en mars 1989 concernant la sauvegarde de la couche d'ozone. À l'issue de la conférence, le Premier ministre britannique d'alors, Margaret Thatcher, donna une conférence de presse. Un journaliste lui posa une question qui commençait par la phrase citée ci-dessus «In your opening remarks...». Voici maintenant la traduction, au sens propre du terme, de cet énoncé:

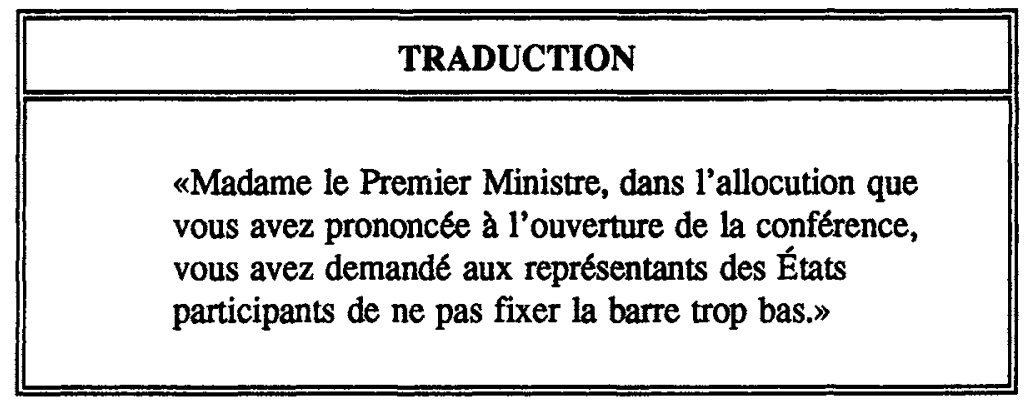

La traduction, entendue dans le vrai sens du terme, s'effectue à la jonction de la connaissance de la langue (ici l'anglais) et de la connaissance des réalités auxquelles renvoie un énoncé. L'exemple cidessus montre à quel point les compléments cognitifs peuvent infléchir 
la signification que l'on reconnaît normalement hors discours aux mots ou locutions d'un système linguistique.

\begin{tabular}{||c|l|l||}
\hline \multicolumn{1}{|c|}{ ORIGINAL } & TRANSCODAGE & \multicolumn{1}{c||}{ DISCOURS } \\
\hline \hline Prime Minister & $\begin{array}{l}\text { Premier Ministre } \\
\text { (homme ou } \\
\text { femme?) }\end{array}$ & $\begin{array}{l}\text { Madame le } \\
\text { Premier Ministre }\end{array}$ \\
\hline opening remarks & $\begin{array}{l}\text { observations limi- } \\
\text { naires }\end{array}$ & $\begin{array}{l}\text { allocution d'ouver- } \\
\text { ture }\end{array}$ \\
\hline $\begin{array}{l}\text { set your eyes too } \\
\text { low }\end{array}$ & $\begin{array}{l}\text { fixer leurs regards } \\
\text { trop bas }\end{array}$ & $\begin{array}{l}\text { fixer la barre trop } \\
\text { bas }\end{array}$ \\
\hline
\end{tabular}

L'enseignement pratique de la traduction professionnelle se situe principalement au niveau de la recherche des équivalences discursives. Il est capital de le rappeler.

\section{L'approche linguistique}

À l'instar de la méthode comparative, l'approche linguistique privilégie la description des langues au moyen cette fois des catégories de la linguistique générale, de la grammaire traditionnelle ou d'une linguistique particulière comme la linguistique transformationnelle. Les manuels de ce genre sont ceux de M. Ballard (1980; 1987), C. Tatilon (1986), H. Chuquet (1987), P. Fournier (1988) et P. Newmark (1988).

Ces ouvrages appliquent à la réflexion sur la traduction un certain nombre de notions de linguistique, de grammaire générale ou de lexicologie. Leurs auteurs ont tendance à communiquer de l'information sur la traduction, sur les actes de parole, sur la sémantique, sur la morphosyntaxe, etc., tout en illustrant leurs propos d'exemples de traduction. Georges Mounin lui-même avait pourtant reconnu que l'ambition de la linguistique à l'égard des traducteurs «est moins de les former que de les informer; moins de leur enseigner leur art, ou de transformer cet art en science infaillible, que de leur fournir sur les phénomènes du langage une culture générale plus large et plus 
complète, qui les éclaire» ${ }^{26}$. Or, le rôle d'un manuel de traduction et d'un cours pratique de traduction n'est-il pas précisément de FORMER des traducteurs?

Certains tenants de l'approche linguistique (Tatilon, Newmark $^{27}$ ) ont tendance à entretenir une vision globale de la traduction. Leurs ouvrages abordent aussi bien les problèmes de la traduction littéraire, poétique, thêâtrale, technique, administrative, publicitaire que la traduction vers le français, l'anglais, l'allemand, l'italien, etc.

Certains de ces ouvrages multiplient aussi les catégories dans un souci, fort louable par ailleurs, de classer les faits de langue ou de traduction. Peter Newmark, par exemple, propose une constellation de catégories et de sous-catégories dans A Textbook of Translation. On y relève pas moins de 15 «translation procedures», 13 «translation methods», 12 «types of neologisms», 18 «types of unfindable words». Pour être véritablement opératoire et avoir une utilité pédagogique, une classification ne doit pas être un simple catalogue de cas d'espèces. L'efficacité pédagogique des exemples est plus ou moins annulée par le pointillisme des observations. En outre, cet émiettement des classifications fait paraître la traduction comme un processus exagérément complexe.

26. «Introduction linguistique aux problèmes de la traduction», pp. 86-87. Les italiques sont de nous.

27. Ces auteurs auraient peut-être préféré voir leur manuel classé sous une rubrique intitulée «approche communicative». Pourtant dans sa préface, G. Mounin écrit à propos de l'auteur de Traduire que «son recours aux connaissances linguistiques les plus actuelles et les plus sûres est solide mais discret» (p. $x$ ). Dans l'index figurent des termes tels que connotation, lexème, oxymoron, pertinence, référent, resémantisation, signifiant, signifié, syntagme, synthème, autant de notions de linguistique générale. La même observation s'applique à l'ouvrage de P. Newmark, A Textbook of Translation. Son index renvoie aux notions de case grammar, collocation, componential analysis et son glossaire définit bon nombre de termes propres à la linguistique. 
En résumé, dans les manuels caractérisés par l'approche linguistique, riches par ailleurs d'excellentes observations sur la traduction, la ligne de démarcation entre la description linguistique et l'enseignement pratique et actif de la traduction n'est pas toujours nette, si l'on définit «enseignement pratique» comme l'acquisition d'un savoirfaire, et non pas seulement comme l'acquisition de connaissances sur la traduction.

\section{Les fiches de travail et cahiers d'exercices}

Les titres de cette subdivision sont les deux cahiers d'exercices de J.-P. Vinay et J. Darbelnet (1968; 1972), celui de V. Watson Rodger (1990) et les ouvrages de F. Grellet (1985; 1991) et A. Duff (1989).

Les deux cahiers d'accompagnement de la Stylistique comparée $d u$ français et de l'anglais proposent des exercices de dissociation des langues dont la présentation suit plus ou moins l'ordre des chapitres du manuel. Peu de textes y figurent. On y trouve, par contre, des fiches sur des mots isolés ou des phrases hors contexte. Il est significatif que dans les programmes de traduction, l'étude de la Stylistique comparée fasse l'objet d'un cours distinct des séminaires pratiques de traduction. On reconnaît ainsi implicitement que ce cours est l'occasion d'un perfectionnement linguistique complémentaire ou préparatoire à l'apprentissage de la traduction.

Pour sa part, Françoise Grellet décrit son initiation à la version anglaise (1985) comme «une série de fiches de travail consacrées aux points qui posent le plus de problèmes aux étudiants. Ce classement n'est fondé sur aucune théorie [...] Ce sont les fautes les plus courantes rencontrées dans des versions d'étudiants qui ont dicté le choix des points traités» ${ }^{28}$. Dans son plus récent ouvrage, Apprendre a traduire, l'auteur apporte la précision suivante: «Les exercices proposés dans ce livre ne constituent pas un cours ou une méthode de traduction mais une typologie d'exercices, c'est-à-dire une classification des divers types

28. "The word against the word". Initiation à la version anglaise, p. 4. 
d'exercices d'entraînement à la traduction. ${ }^{29}$ Pour chaque exercice, l'auteur précise le but visé et les «modalités pratiques» d'application en salle de classe. Ce genre de précisions est rarissime dans les manuels de traduction.

Quant au cahier d'exercices de V. Watson Rodger, il ne renferme aucun texte, mais uniquement des phrases hors contexte d'au plus trois lignes. Il est destiné en premier lieu à l'usage des étudiants anglophones de troisième année inscrits dans les universités canadiennes et vise à préparer «non seulement à mieux traduire mais aussi à mieux écrire et même à mieux parler ${ }^{30}$. Il se compose d'une soixantaine d'exercices (fiches) de traduction vers l'anglais ou le français portant sur des points de langue tels que la mise en relief, les expressions figées, les onomatopées, etc.

Ces professeurs, qui ont produit des instruments de perfectionnement linguistique, se refusent à ériger une frontière trop étanche entre la traduction professionnelle et la traduction scolaire, d'une part, et entre la traduction litteraire et la traduction de textes pragmatiques, d'autre part. Les manuels de F. Grellet et de A. Duff se présentent comme des «resource books» à l'intention des professeurs de langue désireux de varier ou de renouveler le contenu de leurs cours en mettant au menu des exercices de traduction ${ }^{31}$. C'est aux professeurs de choisir dans la gamme des exercices proposés ceux qui conviennent au niveau et aux besoins de leurs étudiants.

29. Apprendre à traduire, p. 14.

30. Apprendre à traduire, p. vii.

31. «Translation is designed to be used by teachers and students from any language background who are involved in the study of English. The aim of the book is to provide the teacher with a wide range of translation activities devised specifically for language learners. The main concern of the book is not how to teach translation, but how to use translation in teaching, as one approach among many in the language class.» A. Duff, Translation, p. 13. 
Ces ouvrages ne sont pas à proprement parler des méthodes de traduction. Apprendre d traduire est un titre quelque peu trompeur. Ces manuels, comme de nombreux autres axés sur l'apprentissage de la langue, refléteraient plus fidèlement leur orientation et leur contenu s'ils étaient coiffés d'un titre tel que Apprendre l'anglais (ou le français) au moyen d'exercices de traduction. Car c'est une chose d'apprendre une langue seconde, c'en est une autre de faire l'apprentissage de la traduction au sens que l'on donne à ce mot dans les écoles professionnelles.

Quoi qu'il en soit, ces exercices de traduction scolaire, correspondant à autant de fiches de travail, peuvent se révéler une source d'inspiration pour les professeurs qui tenteront de concevoir une véritable méthode d'enseignement de la traduction professionnelle.

\section{L'enseignement par objectifs d'apprentissage}

Dans cette dernière catégorie, j’ai regroupé les manuels qui tentent d'organiser l'enseignement de la traduction professionnelle autour d'objectifs clairement définis et ordonnés selon une séquence progressive. Je suis personnellement partisan de cette démarche pédagogique (J. Delisle, 1980; 1989) tout comme mon compatriote C. Bédard $(1986 ; 1987)^{32}$.

Un manuel conçu selon les principes de l'enseignement par objectifs doit obligatoirement limiter ses ambitions et préciser le public visé, son niveau, la langue d'arrivée et le domaine d'étude (traduction générale, technique, administrative, économique, etc.). Il ne peut pas

32. On peut aussi classer dans cette catégorie la thèse de Christine Durieux, Fondement didactique de la traduction technique (Coll. «Traductologie», $n^{0} 3$, Paris, Didier Érudition, 1988, 171 p.) et celle, inédite, de Monique $\mathrm{C}$. Cormier, Traduction technique et pédagogie (thèse de doctorat de $3^{\circ}$ cycle présentée à l'Université de la Sorbonne Nouvelle-Paris III, 1986, 459 p.). Bien que ces deux thèses ne soient pas à proprement parler des «manuels de traduction», elles ne renferment pas moins la description détaillée d'une pédagogie applicable à l'enseignement de la traduction technique. 
prétendre non plus aborder tous les genres de traduction à la fois. Dans la Traduction technique, Claude Bédard écrit: «Notre ouvrage se limite à l'étude de la traduction technique, à l'exclusion de la traduction scientifique. [...] Nous nous sommes aussi borné à la traduction de l'anglais vers le français. ${ }^{33}$ Le Guide d' enseignement qui accompagne cet ouvrage comporte dix-sept objectifs et propose pour chacun d'eux la mise en oeuvre de diverses stratégies pédagogiques: lectures complémentaires, exposés, discussions, exercices variés, études de cas. La diversité de ces procédés a pour but, entre autres, d'agir comme antidote à la monotonie des cours de traduction à vue.

Pour ma part, dans l'Analyse du discours comme méthode de traduction (1980), j'avais pris soin de préciser qu'il s'agissait d'une méthode d'initiation à la traduction professionnelle (et non d'enseignement des langues) qui se limitait «aux messages pragmatiques (non littéraires), généraux (non spécialisés), formulés selon les normes de la langue écrite (non orale), en vue d'un apprentissage dans le sens anglais-français (non l'inverse) $)^{34}$.

Sur le plan théorique, l'enseignement par objectifs d'apprentissage prend appui sur la «théorie interprétative», sans pour autant négliger l'apport important des stylisticiens comparatistes. La démarche contrastive est complémentaire de la démarche interprétative si on en applique les principes et les règles à des textes plutôt qu'à des mots isolés ou à des phrases hors contexte. Sur les plans pédagogique et docimologique, cette méthode cherche à utiliser en enseignement de la traduction la taxinomie de Bloom, Hastings et Madaus ${ }^{35}$.

33. Avant-propos, p. 1

34. L'Analyse du discours comme méthode de traduction, p. 22.

35. Handbook on Formative and Summative Evaluation of Student Learning (New York, McGraw-Hill, 1971). En français, on consultera les traductions de Marcel Lavallée: Taxonomie des objectifs pédagogiques, Tome 1 (1969): Domaine cognitif; Tome 2 (1970): Domaine affectif (Montréal, Éducation nouvelle). Tome 3 (1977): Domaine psychomoteur (Québec, Presses de l'Université du Québec). Voir aussi France Fontaine, Les Objectifs d'apprentissage (Montréal, 
En didactique, un objectif est ce vers quoi tend un apprentissage. Il est formulé en termes de comportements observables et doit prendre en compte le niveau de développement des étudiants, leurs besoins, leurs intérêts. Si un pédagogue n'a pas une idée précise des comportements qu'il souhaite faire acquérir à ses étudiants ou si ces comportements sont énoncés en des termes trop vagues (être capable de traduire des textes économiques), il y a alors de fortes chances qu'il ne sache pas comment structurer son enseignement ni comment mesurer les apprentissages.

L'utilité des objectifs n'est plus à démontrer. On distingue les objectifs généraux et les objectifs spécifiques. Les premiers sont des énoncés d'intention qui tracent dans ses grandes lignes le contenu d'un cours (ou d'un programme). Un cours d'initiation à la traduction générale pourra comporter, par exemple, les objectifs généraux suivants:

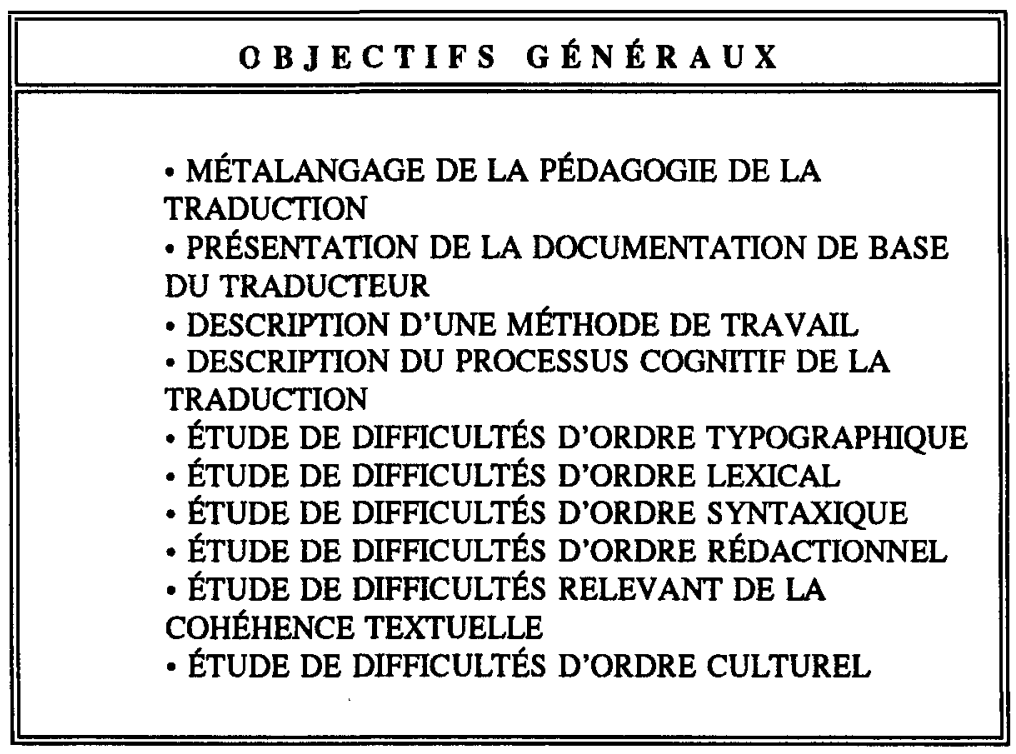

Chacun de ces objectifs généraux pourrait donner lieu à de longs commentaires. Je me contenterai de dire un mot du premier. En

Service pédagogique, Université de Montréal, 1980, 110 p.) 
consultant la cinquantaine de manuels de traduction répertoriés plus haut, j'ai constaté que la plupart des auteurs ne prennent pas toujours la peine de présenter systématiquement le métalangage essentiel à l'apprentissage raisonné de la traduction, et à cet égard, l'Analyse du discours ne fait pas exception. Or, l'expérience prouve qu'il est difficile de tenir un discours réfléchi sur les phénomènes de la traduction ou encore d'évaluer pertinemment des traductions sans disposer d'une terminologie adéquate. Privé de ce cadre notionnel indispensable, on bascule rapidement dans l'impressionnisme stérile contraire à un enseignement universitaire de qualité. Il m'apparaît donc nécessaire de faire acquérir le métalangage de base de la pédagogie de la traduction, qu'il faut se garder de confondre avec les notions de la linguistique générale.

Les objectifs spécifiques sont des énoncés qui décrivent en termes précis ce vers quoi tend l'apprentissage. Ils sont une interprétation concrète des objectifs généraux qui les sous-tendent, et sont formulés en termes de comportements observables. Ainsi, l'objectif spécifique correspondant à l'objectif général «Apprendre le métalangage de la pédagogie de la traduction» pourra s'énoncer de la façon suivante: «L'étudiant devra être en mesure de définir les termes surtraduction, compléments cognitifs, faux comparatif, modulation, interférence, dépersonnalisation du message, etc.» $\AA$ cet objectif viendront s'en greffer d'autres: «L'étudiant devra pouvoir repérer un faux comparatif dans le texte original», "L'étudiant devra pouvoir indiquer quelles sont en français les possibilités de traduction de cette difficulté», «L'étudiant devra pouvoir traduire correctement un faux comparatif relevé dans un texte», etc.

Le rôle des objectifs d'apprentissage est 1) de faciliter la communication (professeur-étudiant/professeur-professeur); 2) de faciliter le choix des instruments pédagogiques; 3) de susciter des activités d'apprentissage et 4) de fournir des bases d'évaluation. Dans le numéro spécial de la revue Meta consacré à l'enseignement de la traduction au Canada, j'ai donné un aperçu de la façon dont on peut organiser un cours d'initiation à la traduction économique selon la 
méthode d'enseignement par objectifs généraux et spécifiques ${ }^{36}$. J'ai la ferme conviction qu'il est tout aussi possible de structurer l'enseignement de la traduction générale autour d'objectifs clairement definis. Le manuel que je prépare actuellement renfermera une douzaine d'objectifs généraux et une soixantaine d'objectifs spécifiques, auxquels s'ajouteront de nombreux exercices et textes d'application.

\section{Conclusion}

Cet essai de classification nous permet de constater que les auteurs des manuels répertoriés ne donnent pas tous le même sens au mot traduction. Sous leur plume, ce terme désigne tout autant le transcodage de mots, syntagmes ou phrases hors contexte que les équivalences discursives. Celles-ci sont établies par un traducteur qui, traduisant un texte «réel», associe des compléments cognitifs aux formes linguistiques du texte afin de réaliser un acte de communication.

La traduction est présente depuis toujours en didactique des langues où elle est un moyen d'analyser et d'assimiler une langue étrangère. Elle est présente aussi dans les écoles professionnelles où, cette fois, elle est une fin en soi. Dans ces unités d'enseignement, on apprend à traduire en vue d'exercer un métier et c'est pourquoi la formation professionnelle qui y est dispensée commande une pédagogie distincte de l'enseignement des langues. Mais qu'est-ce qui distingue cette pédagogie?

Rappelons que toute bonne méthode d'enseignement doit clairement préciser son objet, sérier les difficultés propres au domaine, fixer des objectifs d'apprentissage à atteindre en fonction des compétences à acquérir, préciser les moyens à mettre en oeuvre pour y arriver, établir une progression dans la formation et prévoir les modalités d'évaluation des compétences acquises.

Si l'on admet que la traduction professionnelle peut faire l'objet d'un enseignement sui generis, il faut alors se demander quelles

36. «L'initiation à la traduction économique», Meta, XXXII(2), juin 1988, pp. 204-215. 
sont les compétences particulières que doivent acquérir les futurs traducteurs professionnels. Je pense pouvoir affirmer que les cinq compétences suivantes, définies par Roda P. Roberts ${ }^{37}$, rallieraient la majorité des professeurs des écoles de traduction:

1) linguistique (capacité de comprendre la langue de départ et qualité d'expression de la langue d'arrivée)

2) traductionnelle (capacité de saisir l'articulation du sens dans un texte, de le rendre sans le déformer dans la langue d'arrivée tout en évitant les interférences)

3) méthodologique (capacité de se documenter sur un sujet donné et d'assimiler la terminologie propre au domaine)

4) disciplinaire (capacité de traduire des textes dans quelques disciplines de base, telles que l'économie, l'informatique, le droit)

5) technique (capacité d'utiliser diverses aides à la traduction, telles que traitement de texte, banques de terminologie, machines à dicter, etc.)

Il est frappant de constater que ces compétences sont presque identiques à celles qui figurent dans un ouvrage récent d'un professeur de l'Institut de traduction et d'interprétation de l'Université de Heidelberg, Christiane Nord: «[...] the essential competences required of a translator [are] competence of text reception and analysis, research competence, transfer competence, competence of text production, competence of translation quality assessment, and, of course, linguistic and cultural competence both on the source and the target side $\left[\ldots . .,{ }^{38}{ }^{38}\right.$

On voit que la formation des traducteurs professionnels exige beaucoup plus qu'une formation linguistique, si importante soit-elle. Les

37. "Compétence du nouveau diplômé en traduction», Traduction et Qualité de langue, p. 172.

38. Text Analysis in Translation, p. 235. 
manuels qui leur sont destinés doivent donc refléter cette particularité, ce qui est loin d'être le cas actuellement. L'aspect documentaire, par exemple, absent des manuels d'apprentissage des langues, devrait occuper une place importante dans un manuel conçu pour la formation de traducteurs professionnels. De même, le métalangage de la pédagogie de la traduction devrait permettre de faire la distinction entre «les insuffisances de langues et les erreurs de méthode» ${ }^{39}$.

Nombreuses aussi sont les exigences à respecter concernant le choix des textes, comme le rappelle Christiane Nord:

These texts have to be presented to the students in such a way that as much information as possible is provided on the situation in which the text "functioned" (in the original medium, as a photocopy, with additional details on the sender, his intention, time and place of production etc.). This is why collections of texts which do not specify the sources and even isolated sentences for translation are completely pointless. ${ }^{40}$

Les textes littéraires, très utilisés en enseignement des langues, devraient normalement occuper une place secondaire dans les écoles professionnelles où les textes pragmatiques concourent plus directement à la formation des futurs traducteurs. Il y a en outre une façon d'aborder le texte pragmatique qui differe de la façon d'aborder un texte littéraire. Comme le remarque avec justesse Danica Seleskovitch, elle est «absurde la conviction que les mots d'un texte [pragmatique] sont tous délibérément choisis par leur auteur. Ce qui est encore vrai du poète, du littérateur, ne l'est pas des autres auteurs [...] $\gg^{41}$.

En somme, on peut faire servir les exercices de traduction à des fins les plus diverses, et chacun des 49 manuels énumérés plus haut trouve sans aucun doute son utilité. Mais il n'est pas interdit de

39. Seleskovitch et M. Lederer, Pédagogie raisonnée de l'interprétation, p. 170

40. Text Analysis in Translation, p. 147.

41. «Traduction et traductologie.» [A paraitre.] 
souhaiter, au nom de l'efficacité de l'enseignement, de voir se multiplier les manuels de traduction spécifiquement adaptés à la formation des futurs traducteurs de métier.

\section{Université d'Ottawa}

\section{Références}

BARRA, J. P. (1975). «Quelques considérations sur l'enseignement de la traduction», Taal Taalkunde Vertaalkunde, Gent, Provincie Oost-Vlaanderen, pp. 9-18.

BÉDARD, Claude (1986). La Traduction technique. Principes et pratique, Montréal, Linguatech, $254 \mathrm{p}$.

(1987). Guide d'enseignement de la traduction technique, Montréal, Linguatech, 59 p. +39 pages d'annexes.

BONNEROT, L., et al. (1968). Chemins de la traduction, Paris, Didier, $307 \mathrm{p}$.

GUIDE DU REVISEUR (1985). Ottawa. Bureau de la traduction. Direction générale de la terminologie et de la documentation. Division de la formation et du perfectionnement. Pagination discontinue. 14 annexes. (Document interne. Épuisé.)

DELISLE, Jean (1980). L'Analyse du discours comme méthode de traduction, coll. «Cahiers de traductologie», $n^{\circ} 2$, Ottawa, Les Presses de l'Université, 282 p.

(1988). «L'initiation à la traduction économique», Meta (numéro spécial «L'enseignement de la traduction au Canada»), XXXIII(2), juin, pp. 204-215.

DEMANUELLI Claude et Jean DEMANUELLI (1991). Lire et traduire, Paris, Masson, 241 p.

DUFF, Alan (1989). Translation, Oxford University Press, 160 p. 
GOUADEC, Daniel (1974). Comprendre et traduire, Paris, Bordas, $160 \mathrm{p}$.

GRELLET, Françoise (1991). Apprendre à traduire. Typologie d'exercices de traduction, Nancy, Presses Universitaires de Nancy, $217 \mathrm{p}$.

(1985). «The word against the word». Initiation d la version anglaise, Paris, Hachette, 287 p.

GUILLEMIN-FLESCHER, Jacqueline (1981). Syntaxe comparée du français et de l'anglais. Problemes de traduction, Paris, Éditions Ophrys, 549 p.

HORGUELIN, Paul (1981). Anthologie de la maniere de traduire. Domaine français, Montréal, Linguatech, $230 \mathrm{p}$.

(1985). Pratique de la révision, Montréal, Linguatech, $196 \mathrm{p}$.

(1988). «La révision didactique», Meta (numéro spécial «L'enseignement de la traduction au Canada»), XXXII(2), juin, pp. 253-257.

LAVAULT, Élisabeth (1985). Fonctions de la traduction en didactique des langues, coll. «Traductologie», $\mathrm{n}^{\circ}$ 2, Paris, Didier Érudition, $115 \mathrm{p}$.

MOUNIN, Georges (1976). «Introduction linguistique aux problèmes de la traduction», Linguistique et traduction, Bruxelles, Dessart et Mardaga, pp. 77-88. [Réédition de l'article paru dans Le Français dans le monde, $\mathrm{n}^{\circ} 54,1 / 2,1968$, pp. 13-16.]

(1982). «Pour une pédagogie de la traduction», Cuadernos de traducción $e$ interpretación, (E.U.T.I.), $\mathrm{n}^{\circ} 1$, pp. 11-19.

NEWMARK, Peter (1988). A Textbook of Translation, London/New York, Prentice Hall, 292 p. 
NORD, Christiane (1991). Text Analysis in Translation. Theory, Methodology, and Didactic Application of a Model for Translation-Oriented Text Analysis, Translated from the German by Christiane Nord and Penelope Sparrow, Amsterdam/Atlanta, Éditions Rodopi, 250 p.

POLLAK, Liliane (1989). La Traduction sans peur... et sans reproche, Montréal, Guérin éditeur, $186 \mathrm{p}$.

ROBERTS, Roda P. (1984). «Compétence du nouveau diplômé en traduction», Traduction et Qualité de langue, Actes du colloque, Société des traducteurs du Québec/Conseil de la langue française, Québec, Éditeur officiel du Québec, pp. 172184.

SÉLESKOVITCH, Danica (à paraître). «Traduction et traductologie», communication présentée lors du XII Congrès mondial de la Fédération internationale des traducteurs, Belgrade, août 1990.

et Marianne LEDERER (1989). Pédagogie raisonnée de l'interprétation, Paris, Didier Érudition, coll. «Traductologie», $\mathrm{n}^{\circ} 4,281 \mathrm{p}$.

TATILON, Claude (1986). Traduire. Pour une pédagogie de la traduction, Toronto, Éditions du Gref, coll. «Traduire, Écrire, Lire», $177 \mathrm{p}$.

VAN HOOF, Henri (1989). Traduire l'anglais. Théorie et pratique, Paris, Duculot, 215 p.

(1986). Précis pratique de traduction médicale, Paris, Maloine éditeur, $308 \mathrm{p}$.

VINAY, Jean-Paul et Jean DARBELNET (1958), Stylistique comparée du français et de l' anglais, Paris/Montréal, Didier/Beauchemin, $331 \mathrm{p}$. 
VITALE, Geoffrey, et al. (1978). Guide de la traduction appliquée, t. I, Version, Montréal/Paris, Les Presses de l'Université du Québec/Librairie Vuibert, 397 p.

WATSON RODGER, Valentine (1990). Apprendre d traduire. Cahier d'exercices pour l'apprentissage de la traduction françaisanglais, anglais-français, Toronto, Canadian Scholars' Press Inc., $226 \mathrm{p}$.

WOODSWORTH, Judith (1990). Compte rendu de l'ouvrage de Pamela Russell, How to Write a Précis, TTR, III(1), pp. 131-133. 